\title{
The trade-off between mobility and vaccination for COVID-19 control: a metapopulation modeling approach
}

\author{
Fernando Saldaña ${ }^{\mathrm{a}}$, Jorge X. Velasco-Hernández ${ }^{\mathrm{a}}$ \\ ${ }^{a}$ Instituto de Matemáticas, Campus Juriquilla, 76230, Universidad Nacional Autónoma \\ de México, Quéretaro, Mexico
}

\begin{abstract}
November 2020 received a string of encouraging results from leading vaccine developers raising hopes for the imminent availability of an effective and safe vaccine against the SARS-CoV-2. In the present work, we discuss the theoretical impact of introducing a vaccine across a range of scenarios. In particular, we investigate how vaccination coverage, efficacy, and delivery time affect the control of the transmission dynamics in comparison to mobility restrictions. The analysis is based on a metapopulation epidemic model structured by risk. We perform a global sensitivity analysis using the Sobol method. Our analysis suggest that the reduction of mobility among patches play a significant role in the mitigation of the disease close to the effect of immunization coverage of $30 \%$ achieved in 4 months. Moreover, for an immunization coverage between 20\%-50\% achieved in the first half of 2021 with a vaccine efficacy between $70 \%-95 \%$, the percentage reduction in the total number of SARS-CoV-2 infections is between $30 \%-50 \%$ by the end of 2021 in comparison with the no vaccination scenario.
\end{abstract}

Keywords: COVID-19, SARS-CoV-2, Epidemic model, Disease modeling, Vaccines

Email addresses: fernando.saldana@im.unam.mx (Fernando Saldaña), jx.velasco@im.unam.mx (Jorge X. Velasco-Hernández) 


\section{Introduction}

Even though several countries have implemented social distancing and other non-pharmaceutical interventions to mitigate the SARS-CoV-2 epidemic, the number of infections continues to increase around the world [31]. After eleven months SARS-CoV-2 has infected more than 60 million and killed more than 1.4 million people globally. According to the Johns Hopkins University database, Latin America has been one of the most affected areas with more than 430,000 deaths registered, which represents approximately $30 \%$ of the worldwide total. In particular, Mexico's death rate as a percent of all confirmed cases is around $10 \%$ being one of the highest in the world as of November 20, 2020.

In response to the COVID-19 crisis, there has been an unprecedented collaborative effort by researchers to develop an effective and safe vaccine. There are currently more than 100 COVID-19 vaccine candidates under development, with a number of these in the human trial phase [20]. At the time of writing (November 2020), there has been a string of encouraging results from leading vaccine developers. On November 9, the drug company Pfizer announced that their vaccine (co-developed by BioNTech) was more than $90 \%$ effective at preventing COVID-19 with no serious safety concerns [11]. Two days later, the Russian developers behind the Sputnik V vaccine announced, in a press release, that their vaccine was $92 \%$ effective [10]. Pfizer \& BioNTech results are based on 94 cases, whereas Sputnik V's efficacy was measured with 20 COVID-19 cases. On November 16, the company Moderna announced that their vaccine is more than $94 \%$ effective at preventing COVID-19, based on an analysis of 95 cases [9]. Finally, AstraZeneca and the University of Oxford announced on November 23 that their vaccine has on average $70 \%$ of effectiveness based on data from late-stage clinical trials. Pfizer \& BioNTech and Moderna's vaccines can only be kept outside of ultracold freezers for a few weeks, making their storage and distribution very challenging in regions with poor infrastructure [23] but, unlike the Moderna and Pfizer immunizations, the AstraZeneca-Oxford vaccine does not require a sophisticated cold chain to stay in good condition and is therefore expected to be easier to distribute in developing countries.

As vaccine development continues, there is an urgent need to assess the population-level impact of vaccine introduction $[3,5,7,19]$. The main goal of this work is to investigate the theoretical impact of introducing a vaccine under a number of scenarios. Rather than obtaining quantitative predictions 
of the epidemic course, we explore a spectrum of possibilities to gain qualitative insight on how much the initial introduction of the vaccine can slow the spread of the disease. Many things remain unknown: how effective vaccines will be, the duration of vaccine-induced immunity, and vaccine supply. We particularly concentrate on the impact of vaccine efficacy, coverage, duration of protection, and delivery time on the transmission dynamics of COVID-19. Our analysis is based on a risk-structured metapopulation epidemic model that incorporates mobility foloowing [4]. The risk of acquiring an infection is quantified as a function of the time spent within a particular location where the effective number of contacts that an individual has varies across locations being higher in places with some form of mass gathering e.g. public transportation, malls, religious ceremonies, universities, etc [12]. We exemplify our model with the case of Mexico City.

The paper is structured as follows. In the next section, we formulate our mathematical model and obtain the basic and effective reproduction numbers. In section 3 , we investigate a range of vaccination scenarios that can help to evaluate the population-level impact of a COVID-19 vaccine in a Mexican setting, and in section 4 we analyze the role of mobility and patchdependent risk in the proposed vaccination scenarios. To have a more complete understanding of how vaccine-associated parameters affect the model outcomes, in section 5 we perform a variance-based global sensitivity analysis (GSA) using the Sobol method. Finally, in section 6, we summarize our findings and discuss future work.

\section{Methods}

\subsection{Model formulation}

We presnt a risk-structured metapopulation epidemic model based on an extension of the classical Kermack-McKendrick SEIR model with the approach of Bichara et al. [4]. In this context, metapopulation epidemic models assume that demographic and disease dynamics occur at a comparable timescale, but individuals movement among patches occurs at a faster time scale.

For our model formulation, we consider a 3-patch geographically structured population. Each of the these three patches, labeled $P 1, P 2$, and $P 3$, represents risk areas with low-, middle- and high-risk of infection, respectively. The risk of infection may depend on environmental, socioeconomic, demographic and sanitary conditions [12]. The resident population 
$N_{i}$ of patch $i$, which consist on individuals who normally live in that area, is further sub-divided into eight mutually exclusive compartments of nonvaccinated susceptible $\left(S_{i}\right)$, vaccinated susceptible $\left(V_{i}\right)$, exposed $\left(E_{i}\right)$, vaccinated exposed $\left(\tilde{E}_{i}\right)$, asymptomatic infectious $\left(A_{i}\right)$, symptomatic infectious $\left(I_{i}\right)$, reported infectious $\left(I_{i}^{r}\right)$ and recovered $\left(R_{i}\right)$, hence $N_{i}=S_{i}+V_{i}+E_{i}+$ $\tilde{E}_{i}+A_{i}+I_{i}+I_{i}^{r}+R_{i}$ with $i=1,2,3$.

Movement among patches is described using the residence time matrix $\mathbf{P}=\left[p_{i j}\right], i, j=1,2,3$, where $p_{i j}$ represents the time $i$-residents spend in $j$-environments, with $\sum_{j=1}^{3} p_{i j}=1$ for all $i . N_{j}^{e}$ is the effective population of patch $j$ at time $t$, that is, the number of individuals who are physically present in patch $j$ at time $t$, then

$$
N_{j}^{e}=\sum_{k=1}^{3} p_{k j} N_{k} .
$$

This notation is also used for the disease compartments, for example, $I_{j}^{e}=$ $\sum_{k=1}^{3} p_{k j} I_{k}$ is the effective infectious population in patch $j$ at time $t$.

The model is given by the following system of differential equations

$$
\begin{aligned}
& \frac{d S_{i}}{d t}=b_{i} N_{i}-\sum_{j=1}^{3} \beta_{j} p_{i j} S_{i} \frac{I_{j}^{e}+\alpha_{j} A_{j}^{e}}{N_{j}^{e}}-u_{i} S_{i}+w_{i} R_{i}+\theta_{i} V_{i}-d_{i} S_{i}, \\
& \frac{d V_{i}}{d t}=u_{i} S_{i}-\sum_{j=1}^{3}\left(1-\psi_{i}\right) \beta_{j} p_{i j} V_{i} \frac{I_{j}^{e}+\alpha_{j} A_{j}^{e}}{N_{j}^{e}}-\theta_{i} V_{i}-d_{i} V_{i}, \\
& \frac{d E_{i}}{d t}=\sum_{j=1}^{3} \beta_{j} p_{i j} S_{i} \frac{I_{j}^{e}+\alpha A_{j}^{e}}{N_{j}^{e}}-k_{i} E_{i}-d_{i} E_{i}, \\
& \frac{d \tilde{E}_{i}}{d t}=\sum_{j=1}^{3} \beta_{j} p_{i j}\left(\left(1-\psi_{i}\right) V_{i}\right) \frac{I_{j}^{e}+\alpha A_{j}^{e}}{N_{j}^{e}}-k_{i} E_{i}-d_{i} E_{i}, \\
& \frac{d A_{i}}{d t}=\left(1-\rho_{i}\right) k_{i} E_{i}+\left(1-\tilde{\rho}_{i}\right) k_{i} \tilde{E}_{i}-\gamma_{i}^{a} A_{i}-d_{i} A_{i}, \\
& \frac{d I_{i}}{d t}=\rho_{i} k_{i} E_{i}+\tilde{\rho}_{i} k_{i} \tilde{E}_{i}-\gamma_{i} I_{i}-\nu_{i} I_{i}-d_{i} I_{i}, \\
& \frac{d I_{i}^{r}}{d t}=\nu_{i} I_{i}-\gamma_{i}^{r} I_{i}^{r}-d_{i} I_{i}^{r}, \\
& \frac{d R_{i}}{d t}=\gamma_{i}^{a} A_{i}+\gamma_{i} I_{i}+\gamma_{i}^{r} I_{i}^{r}-w_{i} R_{i}-d_{i} R_{i}, \quad i=1,2,3 .
\end{aligned}
$$


The parameters $b_{i}$ and $d_{i}$ represent the per capita birth and death rates in patch $i$. Susceptible individuals are vaccinated at a rate $u_{i}$ and acquire the infection after an effective contact with a symptomatic infectious person with an effective contact rate $\beta_{j}$ that is an index of the patch-specific risk $(j=$ $1,2,3)$. The parameter $0<\alpha_{i}<1$ measures the relative infectiousness of the asymptomatic infectious class in relation to symptomatic individuals in patch $i(i=1,2,3)$. The vaccine reduces the force of infection (FOI) by a factor $\psi_{i}$ with $0<\psi_{i}<1$. The parameter $1 / k_{i}$ represents the mean latent period; after this, a fraction $\rho_{i}$ of the exposed class $E_{i}$ transition to the symptomatic infectious class $I_{i}$, while the other fraction $1-\rho_{i}$ enter the asymptomatic infectious class $A_{i}$. The parameter $\tilde{\rho}_{i}$ represents the symptomatic fraction in the vaccinated exposed class $\tilde{E}_{i}(i=1,2,3)$ with $\tilde{\rho} \leq \rho$. Individuals in the symptomatic infectious class are reported at a rate $\nu_{i}$ and are effectively isolated and they no longer contribute to the FOI. The parameters $\gamma_{i}, \gamma_{i}^{a}$ and $\gamma_{i}^{r}$ are the recovery rates for classes $I_{i}, A_{i}$, and $I_{i}^{r}$ respectively $(i=1,2,3)$.

Note that in our model, vaccination not only may prevent SARS-CoV2 infection but also it may prevent the symptomatic disease COVID-19. Moreover, unlike previous COVID-19 epidemic models (see, for example, $[7,16,21,22,28,32])$ system (2) allows the possibility of reinfections. This is essential since it is not yet known how long natural immunity will last and there have been already confirmed cases of coronavirus reinfection [15]. Furthermore, it is also uncertain whether vaccine-induced immunity will be short- or long-lived; therefore, we assume natural and vaccine-induced immunity are loss at rates, $w_{i}$ and $\theta_{i}$, respectively $(i=1,2,3)$.

Since we look at the dynamics of model (2) over a relatively short time interval, we assume a constant population size with the birth rate equal to the death rate, that is, $b_{i}=d_{i}$. Therefore, the resident population in patch $i$ is a constant $N_{i}^{*}(i=1,2,3)$. The boundedness, positiveness, and continuity of the solutions are fairly straightforward to obtain from the model equations and the fixed population size assumption. Hence, system (2) is mathematically and epidemiologically well-posed [18].

Note that patch $i$ always has a disease-free equilibrium of the form

$E_{0}^{i}=\left(S_{i}^{*}, V_{i}^{*}, E_{i}^{*}, \tilde{E}_{i}^{*}, A_{i}^{*}, I_{i}^{*}, I_{i}^{r *}, R_{i}^{*}\right)=\left(\frac{\left(\theta_{i}+d_{i}\right) b_{i} N_{i}^{*}}{d_{i}\left(u_{i}+\theta_{i}+d_{i}\right)}, N_{i}^{*}-S_{i}^{*}, 0,0,0,0,0,0\right)$.

The 3-patch metapopulation epidemic model (2) is at the disease-free equilibrium if every patch is at the disease-free equilibrium. 


\subsection{The basic reproduction number}

In the simple case in which patches are isolated from the others, that is, $p_{i j}=\delta_{i j}$, where $\delta_{i j}$ is the Kronecker delta $(i, j=1,2,3)$; the patch-specific basic reproduction numbers are easily obtained,

$$
\mathcal{R}_{0}^{i}=\left(1-\rho_{i}\right) T_{A}^{i}+\rho_{i} T_{I}^{i}, \quad i=1,2,3,
$$

where $T_{A}^{i}=\alpha_{i} \beta_{i} k_{i} /\left(k_{i}+d_{i}\right)\left(\gamma_{i}^{a}+d_{i}\right)$ and $T_{I}^{i}=\beta_{i} k_{i} /\left(k_{i}+\nu_{i}+d_{i}\right)\left(\gamma_{i}+d_{i}\right)$, measure the contributions of the asymptomatic and symptomatic infectious classes to the production of new infections, respectively. The patch-specific basic reproduction number is a threshold quantity and $\mathcal{R}_{0}^{i}<1$ implies the local stability of $E_{0}^{i}$. However, we have to remark that this result is only local and there exist the possibility that the introduction of an imperfect vaccine in system (2) can lead to the emergence of a backward bifurcation [17].

To compute the basic reproduction number in the presence of movement, we take a next-generation approach [14] using the method of [30]. Ordering the infected subsystem $\left(I_{i}^{r}\right.$ class can be omitted because they do not contribute to the FOI) as

$$
E_{1}, A_{1}, I_{1}, E_{2}, A_{2}, I_{2}, E_{3}, A_{3}, I_{3}
$$

we obtain a block matrix $\mathbf{F}=\left[\tilde{\mathbf{F}}_{i j}\right]$, where for $i, j=1,2,3, \tilde{\mathbf{F}}_{i j}$ is an $3 \times 3$ matrix with

$$
\tilde{\mathbf{F}}_{i j}=\left[\begin{array}{ccc}
0 & \lambda_{i j}^{a} & \lambda_{i j} \\
0 & 0 & 0 \\
0 & 0 & 0
\end{array}\right], \lambda_{i j}^{a}=\sum_{k=1}^{3} \alpha_{k} \beta_{k} \frac{p_{i k} p_{j k} N_{k}^{*}}{N_{k}^{e *}}, \lambda_{i j}=\sum_{k=1}^{3} \beta_{k} \frac{p_{i k} p_{j k} N_{k}^{*}}{N_{k}^{e *}}
$$

The matrix $\mathbf{V}$ is a block diagonal matrix $\mathbf{V}=\operatorname{diag}\left(\tilde{\mathbf{V}}_{i i}\right)$, where for $i=1,2,3$, $\tilde{\mathbf{V}}_{i i}$ is an $3 \times 3$ matrix with

$$
\tilde{\mathbf{V}}_{i i}=\left[\begin{array}{ccc}
k_{i}+d_{i} & 0 & 0 \\
-\left(1-\rho_{i}\right) k_{i} & \gamma_{i}^{a}+d_{i} & 0 \\
-\rho_{i} k_{i} & 0 & \gamma_{i}+d_{i}
\end{array}\right]
$$

The next-generation matrix is the block matrix $\mathbf{K}=\mathbf{F} \mathbf{V}^{-\mathbf{1}}=\left[\tilde{\mathbf{F}}_{i j} \mathbf{V}_{j j}^{-1}\right]$, $i, j=1,2,3$, and the basic reproduction number in the presence of movement is $\mathcal{R}_{0}=\rho(\mathbf{K})$, where $\rho(\cdot)$ is the spectral radius. Note that by definition $\mathcal{R}_{0}$ assumes a fully susceptible population and, hence, control measures, such as 
mass vaccination, that reduce the the number of susceptible individuals in the population technically do not affect the value of $\mathcal{R}_{0}$ [13].

The same method allows us to obtain the effective reproduction number $\mathcal{R}_{e}$ as the spectral radius of the controlled next-generation matrix $\mathbf{K}_{c}=$ $\mathbf{F}_{c} \mathbf{V}_{c}^{-1}$, where $\mathbf{F}_{c}=\left[\tilde{\mathbf{F}}_{i j}^{c}\right]$, where for $i, j=1,2,3, \tilde{\mathbf{F}}_{i j}^{c}$ is an $4 \times 4$ matrix with

$$
\begin{array}{r}
\tilde{\mathbf{F}}_{i j}^{c}=\left[\begin{array}{cccc}
0 & 0 & \varphi_{i j}^{a} & \varphi_{i j} \\
0 & 0 & \chi_{i j}^{a} & \chi_{i j} \\
0 & 0 & 0 & 0 \\
0 & 0 & 0 & 0
\end{array}\right], \\
\varphi_{i j}^{a}=\sum_{k=1}^{3} \alpha_{k} \beta_{k} \frac{p_{i k} p_{j k} S_{k}^{*}}{N_{k}^{e *}}, \varphi_{i j}=\sum_{k=1}^{3} \beta_{k} \frac{p_{i k} p_{j k} S_{k}^{*}}{N_{k}^{e *}}, \\
\chi_{i j}^{a}=\sum_{k=1}^{3} \alpha_{k} \beta_{k}\left(1-\psi_{k}\right) \frac{p_{i k} p_{j k} V_{k}^{*}}{N_{k}^{e *}}, \chi_{i j}=\sum_{k=1}^{3} \beta_{k}\left(1-\psi_{k}\right) \frac{p_{i k} p_{j k} V_{k}^{*}}{N_{k}^{e *}} .
\end{array}
$$

The matrix $\mathbf{V}_{c}$ is a block diagonal matrix $\mathbf{V}_{c}=\operatorname{diag}\left(\tilde{\mathbf{V}}_{i i}^{c}\right)$, where for $i=$ $1,2,3, \tilde{\mathbf{V}}_{i i}^{c}$ is an $4 \times 4$ matrix with

$$
\tilde{\mathbf{V}}_{i i}^{c}=\left[\begin{array}{ccccc}
k_{i}+d_{i} & 0 & 0 & 0 & \\
0 & k_{i}+d_{i} & 0 & 0 & \\
-\left(1-\rho_{i}\right) k_{i} & -\left(1-\tilde{\rho}_{i}\right) k_{i} & \gamma_{i}^{a}+d_{i} & 0 & 0 \\
-\rho_{i} k_{i} & -\tilde{\rho}_{i} k_{i} & 0 & \gamma_{i}+d_{i} &
\end{array}\right]
$$

\subsection{Model parameters}

We retrieved the baseline values for some of our model parameters using COVID-19 epidemic data from the Mexican Federal Health Secretary [27] (Secretaría de Salud Mexico) and estimations from previous studies with data from Mexico City [2, 21, 25]. Evidence suggests that about four in five people infected with SARS-CoV-2 develop symptoms [6], therefore, we set $\phi_{i}=0.8$. The mean incubation period for COVID-19 range from 2-14 days, we choose the estimation $1 / k_{i}=5.99$ days [2]. The mean recovery rates for the asymptomatic, symptomatic and reported infectious classes are $\gamma_{i}^{a}=1 / 14, \gamma_{i}=1 / 10.81$, and $\gamma_{i}^{r}=1 / 5.0$ days $^{-1}$, respectively. The average progression rate from the symptomatic-infectious class to the reported infectious class is $\nu_{i}=1 / 3.0$ days $^{-1}$ [21]. The relative infectiousness of the asymptomatic infectious class in relation to symptomatic individuals is $\alpha_{i}=0.45$ [8]. The life expectancy of the Mexican population is roughly 70 years; hence, 
$d_{i}=1 /(70 * 365)$ days $^{-1}$. The results presented in [15] suggest that reinfections are more likely to occur at 12 months after infection. For this study, we assume natural immunity last a year i.e. $1 / w_{i}=365$ days unless otherwise stated. These epidemiological parameters are common for each of the three patches $P 1, P 2, P 3$, since they are geographical regions that comprise people of all ages. The value of the basic reproduction number for COVID-19 in Mexico has been estimated to be between 2.95 and 5.89 with a mean value of 3.87 [2]. Using these estimations, we obtain the following values for the transmission coefficients: $\beta_{1}=0.31, \beta_{2}=0.40$, and $\beta_{3}=0.53$. The initial time for our study is November 20, 2020, and the time horizon to study the effects of vaccination is 365 days. The initial conditions are fixed according to the epidemiological data on November 20 [27]. Since the risk distribution is constantly changing as measure by the number of active cases per geographical area, we simplify assuming that low-risk areas are most frequent than high-risk areas. In particular, we assume a distribution of $50 \%$ in $P 1$, $30 \%$ in $P 2$, and $20 \%$ in $P 3$.

\section{COVID-19 vaccination scenarios}

In this section, we investigate several vaccination scenarios to evaluate the population-level impact of a COVID-19 vaccine. Each scenario is based on a set of numerical values for the vaccine-associated parameters $\Theta=\left\{\left(\tilde{\rho}_{i}, \psi_{i}, \theta_{i}, C_{i}, T_{i}\right), i=1,2,3\right\}$ where $C_{i}$ is the target immunization coverage and $T_{i}$ the target time to achieve that coverage. The vaccination rate $u_{i}$ is obtained from the approximation $1-\exp \left(-u_{i} T_{i}\right)=C_{i}$ [1]. To formulate our scenarios we take into consideration the currently available information of the COVID-19 vaccine candidates [20]. The expected vaccine efficacy is between $70 \%-95 \%$ and the duration of vaccine-induced immunity should be at least 6 months. There is still no reliable information on the proportion of symptomatic infections in vaccinated individuals so for simplicity, we assume this proportion is less than $50 \%$. Besides, the Mexican public health authorities expect to achieve immunization coverage between $10 \%$ and $50 \%$ in the year 2021 [24]. Under these considerations, we propose three scenarios (see Table 1) attempting to reflect a range of possibilities between worst-case and optimistic conditions. Each scenario is further subdivided according to the time needed to reach the target immunization coverage $T_{i}$ : (a) 1 month, (b) 3 months, and (c) 5 months.

Moreover, considering that it is not yet known how long natural immunity 


\begin{tabular}{l|c|c|c|c}
\hline Parameter & Range & Scenario 1 & Scenario 2 & Scenario 3 \\
\hline$C_{i}$ & {$[10 \%, 50 \%]$} & $30 \%$ & $40 \%$ & $50 \%$ \\
$\psi_{i}$ & {$[70 \%, 95 \%]$} & $70 \%$ & $80 \%$ & $90 \%$ \\
$\tilde{\rho}_{i}$ & {$[0 \%, 50 \%]$} & $50 \%$ & $30 \%$ & $10 \%$ \\
$\theta_{i}^{-1}$ & {$[180,365]$ days } & 180 & 250 & 365 \\
\hline \multicolumn{2}{r}{$T_{i} \in[1,5]$ months: (a) 1 month (b) 3 months (c) 5 months } \\
\hline
\end{tabular}

Table 1: Description of the parameter values for the three COVID-19 vaccination scenarios. Each vaccination scenario is further subdivided according to the time needed to reach the target vaccination coverage: (a) 1 month, (b) 3 months, and (c) 5 months. The parameters are the same for all patches $(i=1,2,3)$ and the vaccination rate $u_{i}$ is obtained from the approximation $1-\exp \left(-u_{i} T_{i}\right)=C_{i}$, where $C_{i}$ is the target coverage and $T_{i}$ the time wished to achieve coverage $C_{i}$.

might last and how common reinfection is [15]; we investigate each of the vaccination scenarios for a duration of protective immunity of 180 and 365 days. For all cases, the introduction of the vaccine starts on January 1, 2021. Parameter values that vary among the vaccination scenarios are listed in Table 1, while parameter values common to all scenarios are described in Section 2.3. We start our simulations in the simple case in which individuals spend the same time in all patches; hence, the residence time matrix is $\mathbf{P}_{1 / 3}=$ $\left[p_{i j}\right]$ with $p_{i j}=1 / 3$ for all $i, j$. For this mobility matrix and the baseline parameter values, the value of the basic reproduction number (which do not considers vaccination) is $\mathcal{R}_{0}=1.29$.

Fig. 1 shows the cumulative number of reported cases per day for the proposed vaccination scenarios in Table 1 according to the time needed to achieve the target vaccination coverage: (a) 1 month, (b) 3 months, and (c) 5 months. The no vaccination case is shown in red and the data corresponds to the official cumulative confirmed cases in Mexico City until November 20, 2020. The assumed duration of natural immunity is 180 days, therefore, the number of reported cases in the no vaccination scenario increases rapidly reaching more than 500,000 cases by the end of 2021. In the most optimistic scenario, Scenario 3 (a), the introduction of the vaccine allows maintaining the cumulative reported cases around 300,000 cases by the end of 2021, achieving more than $40 \%$ reduction in the reported cases. For Scenario 3 (a), the effective reproduction number is $\mathcal{R}_{e}=0.59$ so vaccination decreases significantly (more than $50 \%$ ) the value of $\mathcal{R}_{0}$. Fig. 2 also shows the cumulative number of reported cases. Yet, in this case, the assumed duration of natural 
immunity is 365 days and, hence, in the no control case, the reported cases by the end of 2021 is around 420,000 cases. From Fig. 2, we see that if the duration of natural immunity is one year, the introduction of a vaccine with about $90 \%$ of effectiveness (Scenario 3) allows controlling the infection before the end of 2021. However, this is not the case if natural immunity lasts only half a year (see, for example, Scenario 1(c) in Fig. 1). For all the scenarios explored, the value of the effective reproduction number ranges between 0.59 and 1.13. Hence the effect of vaccination on the reduction of the $\mathcal{R}_{0}$ values varies significantly for different conditions. However, we have to remark that we did not consider the effect of non-pharmaceutical interventions which play a significant role in the control of $\mathcal{R}_{e}$.

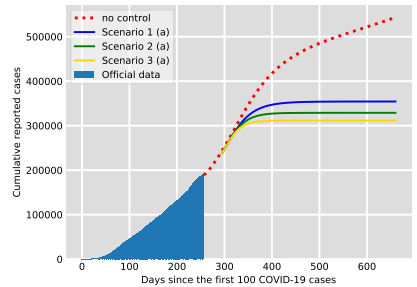

(a)

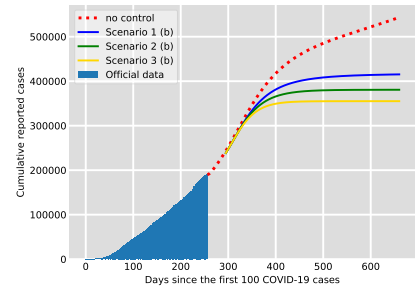

(b)

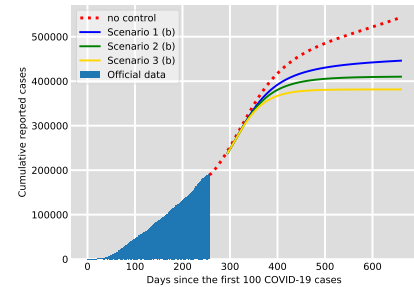

(c)

Figure 1: Cumulative number of reported cases per day for the vaccination scenarios in Table 1 according to the time needed to achieve the target vaccination coverage: (a) 1 month, (b) 3 months, and (c) 5 months. The data corresponds to the official cumulative confirmed cases in Mexico City until November 20, 2020. The assumed duration of natural immunity is 180 days.

\section{Residence times and patch-dependent risk}

\subsection{The role of mobility}

Here, we investigate the role of mobility and patch-dependent risk in the proposed vaccination scenarios. The description of movement among regions is a matter of particular interest for the study of infectious disease transmission among spatially distinct populations and some studies had described the movement between two patches by relationships of the form $p_{i j} \propto N_{i} C_{j} N_{j} f(d)$, where $N_{i}$ is the respective resident population in patch $i$, $C_{i}$ is the population who work in patch $i$ (regardless of their resident patch) and $f(d)$ is a distance kernel [29]. 


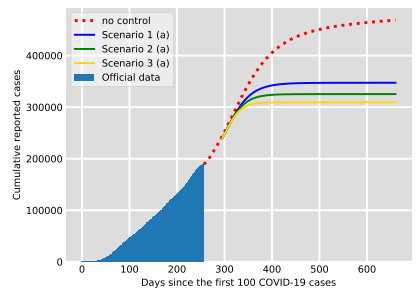

(a)

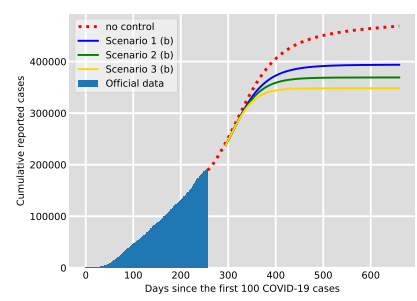

(b)

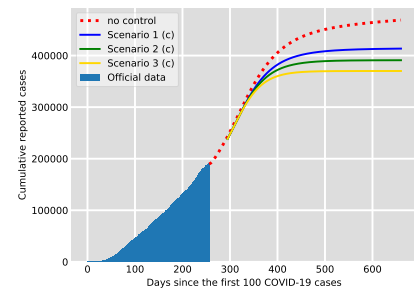

(c)

Figure 2: Cumulative number of reported cases per day for the vaccination scenarios in Table 1 according to the time needed to achieve the target vaccination coverage: (a) 1 month, (b) 3 months, and (c) 5 months. The data corresponds to the official cumulative confirmed cases in Mexico City until November 20, 2020. The assumed duration of natural immunity is 365 days.

System (2) models constant human mobility through the resident time matrix $\mathbf{P}$ and do not captures behavioral responses to disease dynamics that may optimize an index of well-being. Although this effect can be captured placing appropriate restrictions on the entries of $\mathbf{P}[12]$, we limit our study to the case where there is no behavioral change but we propose two mobility matrices (in addition to $\mathbf{P}_{1 / 3}$ ) that may be useful to support public health preparedness. (I) Individuals spend a considerable amount of time in highrisk areas. The residence time matrix for this case is $\mathbf{P}_{I}=\left[p_{i j}\right]$ with $p_{i 3}=0.7$ for all $i$, and $p_{i j}=0.15$ with $i=1,2,3, j=1,2$. (II) There is reduced mobility and individuals stay in their home patch most of their time. The residence time matrix for this case is $\mathbf{P}_{I I}=\left[p_{i j}\right]$ with $p_{i j}=0.8$ with $i=j$, and $p_{i j}=0.1$ with $i \neq j$. All the results are summarized in the plots appearing in Fig. 3. The parameter values are fixed according to Scenario 2 (b) in Table 1 which represents an intermediate case between worst-case and optimistic conditions.

The left column in Fig. 3 shows the number of individuals in the infected classes per patch for the residence time matrix $\mathbf{P}_{1 / 3}$, whereas the middle row shows the results for $\mathbf{P}_{I}$, and the left column the results for $\mathbf{P}_{I I}$. Observe that the prevalence of the infection is, on average, higher for the residence time matrix $\mathbf{P}_{I}$ for all the infected classes in comparison with the other mobility matrices. Moreover, the residence time matrices also have an important influence on the reproduction numbers. The patch specific reproduction numbers (which do not consider movement) for the parameter values chosen 
medRxiv preprint doi: https://doi.org/10.1101/2020.12.09.20246538; this version posted December 11, 2020. The copyright holder for this preprint (which was not certified by peer review) is the author/funder, who has granted medRxiv a license to display the preprint in perpetuity.

It is made available under a CC-BY-NC-ND 4.0 International license .
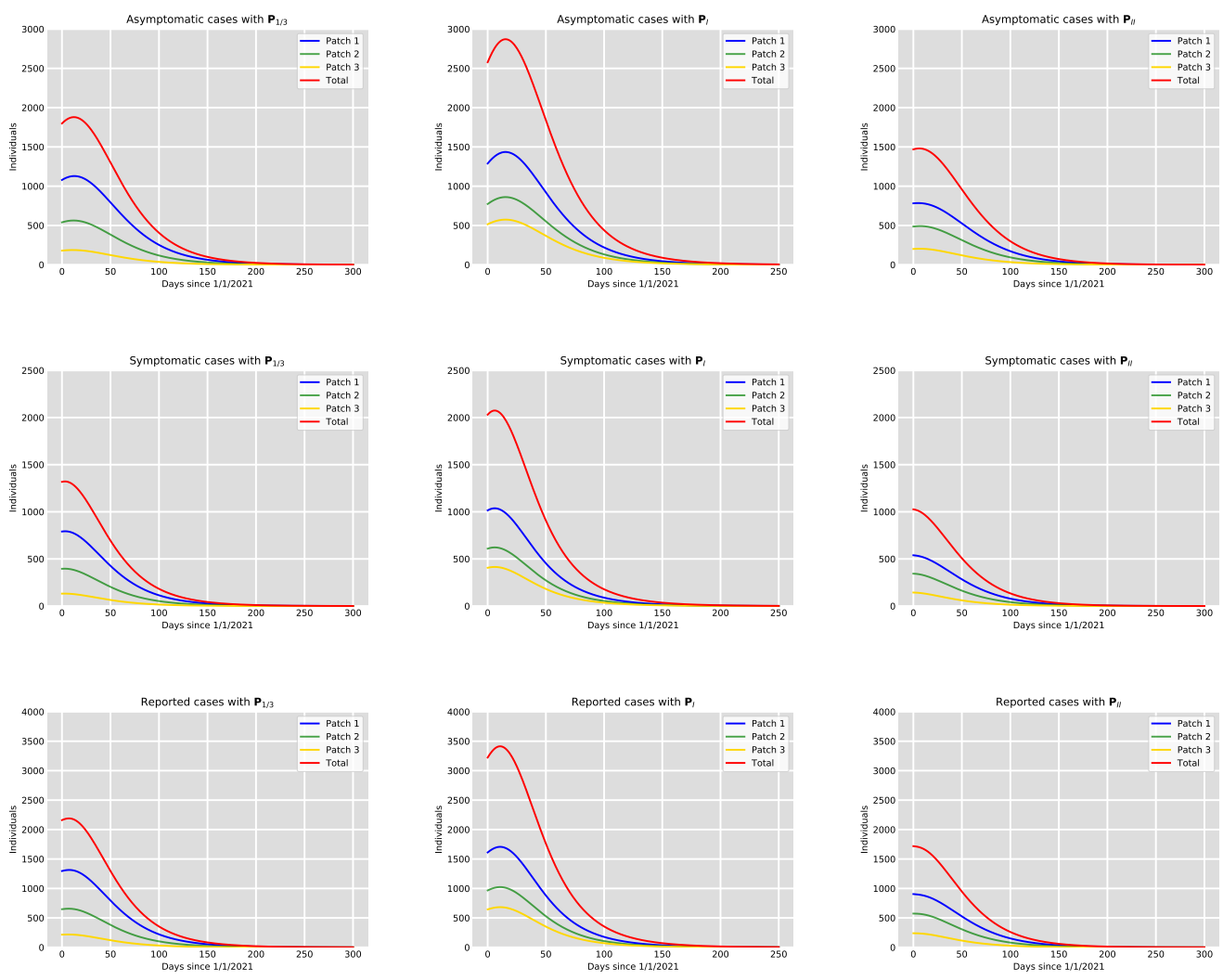

Figure 3: Top row: Asymptomatic cases per patch. Middle row: Symptomatic cases per patch. Low row: Reported cases per patch. Left column: The mobility matrix is $\mathbf{P}_{1 / 3}$. Middle column: The mobility matrix is $\mathbf{P}_{I}$. Right column: The mobility matrix is $\mathbf{P}_{I I}$. For all cases, the parameter values are fixed according to Scenario 2 (b) in Table 1. The simulations start on January 1, 2021, with an initial condition obtained from the results of model (2) simulated from November 20, 2020, until December 31, 2020, depending on the residence time matrix for each case. The assumed duration of natural immunity is 365 days. 
are $\mathcal{R}_{0}^{1}=1.02, \mathcal{R}_{0}^{2}=1.32$, and $\mathcal{R}_{0}^{3}=1.75$. In the presence of movement, the basic and effective reproduction numbers are $\mathcal{R}_{0}=1.12$ and $\mathcal{R}_{e}=0.68$, $\mathcal{R}_{0}=1.5$ and $\mathcal{R}_{e}=0.91, \mathcal{R}_{0}=1.11$ and $\mathcal{R}_{e}=0.67$, for the matrices $\mathbf{P}_{1 / 3}, \mathbf{P}_{I}$ and $\mathbf{P}_{I I}$, respectively. Therefore, if patches are strongly connected, the highrisk patch acts like a source patch promoting an increase in the transmission and the value of the reproduction numbers of the whole model. On the other hand, restricted mobility helps to maintain control of disease transmission. In particular, one can observe that the prevalence levels for the high mobility matrix $P_{I}$ are almost twice the levels for the restricted mobility matrix $P_{I I}$. The disease is eradicated in all cases because the effective reproduction number, that depends on the successful deployment, coverage and efficacy of the vaccine, is less than unity .

\subsection{Vaccination prioritization for high-risk areas}

In previous simulations, we have considered equal vaccination rates for all patches. Nevertheless, long periods of residence in the high-risk patch promote an increase in transmission (see Fig. 3). Hence, it is logical to expect that prioritizing the introduction of the vaccine in the high-risk patch can help mitigate the spread of the epidemic more effectively at least when there is high mobility. Furthermore, if there is a limited vaccine supply, it may be expected that groups in the most affected areas may be recommended to get a COVID-19 vaccine first. What is more, despite the availability of the vaccine, there can be delays in acceptance or even refusal of vaccination in some groups of the population causing a low vaccine coverage in some areas. Taking this into consideration, we consider a scenario in which the highrisk patch has higher immunization coverage and faster deployment of the vaccine in comparison with the other two patches. For illustration purposes, we assume that public health authorities achieve $70 \%$ vaccination coverage after one month of the introduction of the vaccine in patch $P 3$. We maintain patches $P 1$ and $P 2$ under the same conditions used in Section (a) (40\% coverage in three months). We perform simulations to investigate if there is a reduction in the prevalence in comparison with the case of equal vaccination rates for the mobility matrix $\mathbf{P}_{I}$. The results depicted in Fig. 4 imply that prioritizing the high-risk patch reduces the transmission levels, especially in that patch. Nevertheless, this reduction appears to be very small in the other patches. This may be because the high-risk patch comprises less area than the other two patches and therefore high coverage in this patch is not enough to see a considerable reduction in the rest of the patches. 


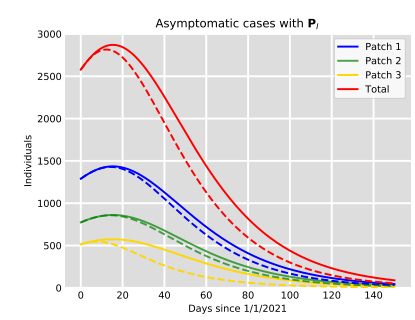

(a)

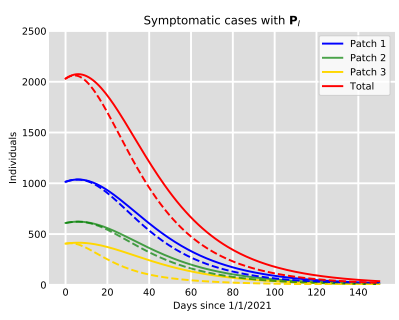

(b)

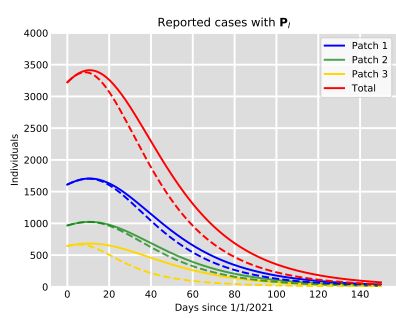

(c)

Figure 4: Dynamics of the infected classes per patch for equal vaccination rates (solid lines) in all patches and vaccine prioritization (dotted lines) for the high-risk patch $P 3$. (a) Asymptomatic classes $A_{1}$ (blue), $A_{2}$ (green), $A_{3}$ (yellow). (b) Symptomatic classes $I_{1}$ (blue), $I_{2}$ (green), $I_{3}$ (yellow). (c) Reported classes $I_{1}^{r}$ (blue), $I_{2}^{r}$ (green), $I_{3}^{r}$ (yellow). The mobility matrix is $\mathbf{P}_{I}$.

\subsection{Coverage, efficacy and delivery time versus mobility}

From the simulations shown in Fig. 4 one can see that even when prioritizing vaccination in the high-risk patch $\left(C_{3}>C_{1}, C_{2}\right.$ and $\left.T_{3}<T_{1}, T_{2}\right)$ reduces the prevalence level, this reduction seems to be still less than the reduction achieved if there is restricted mobility (see the right column in Fig. 3). Therefore, we further explore how vaccination coverage, efficacy, and delivery time affect the control of the transmission dynamics in comparison with the effect of mobility restrictions.

Fig. 5 presents the results. For all plots, the total number of cumulative reported cases for the high-mobility case (the residence time matrix is $\mathbf{P}_{I}$ ) are presented by the red solid lines and red dotted lines represent the low-mobility case (the residence time matrix is $\mathbf{P}_{I I}$ ). These cumulative reported cases are obtained without vaccination and indicate that the expected number of reported infections for Mexico City is between 430,000 cases and 550,000 by the end of 2021 depending on the level of mobility. In Fig. 5 (a), we vary the vaccination coverage between $20 \%-40 \%$ with fixed efficacy at $80 \%$ and delivery time in 5 months. In Fig. 5 (b), we vary vaccine efficacy between $70 \%-90 \%$ with fixed $30 \%$ coverage achieved in 5 months. In Fig. 5 (c), we vary delivery time between 2-6 months for fixed $80 \%$ efficacy and $30 \%$ coverage. The simulations (solid lines) are obtained for the high-mobility matrix $\mathbf{P}_{I}$. From Fig. 5 (a) we see that an efficacy of $80 \%$, with $40 \%$ coverage achieved in 5 months has a similar effect in comparison with restricted mobility without vaccination. In Fig. 5 (b) one can see that even with $90 \%$ vaccine 


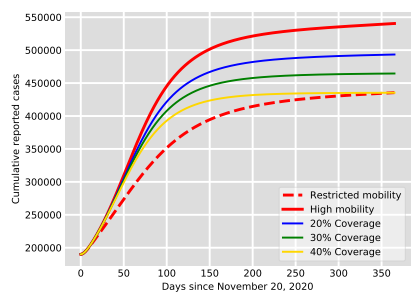

(a)

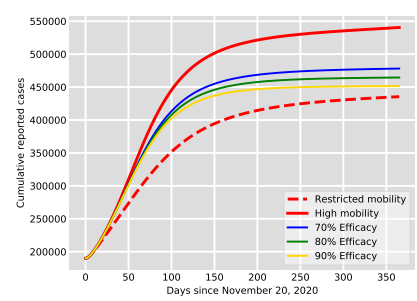

(b)

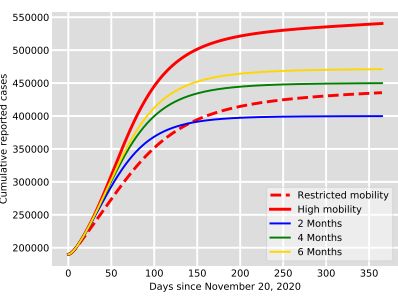

(c)

Figure 5: Impact of coverage, efficacy and delivery time on the cumulative number of reported cases in comparison with the impact of mobility. (a) Vaccination coverage between $20 \%-40 \%$ with fixed efficacy at $80 \%$ and delivery time in 5 months. (b) Vaccine efficacy between $70 \%-90 \%$ with fixed $30 \%$ coverage achieved in 5 months. (c) Delivery time between 2-6 months for fixed $80 \%$ efficacy and 30\% coverage. The proposed scenarios are presented in solid lines (blue, green, yellow) and are obtained for the residence time matrix $\mathbf{P}_{I}$. For all subfigures, the total number of cumulative reported cases for the residence time matrix $\mathbf{P}_{I}$ ) are presented by the red solid lines and red dotted lines represent the low-mobility case where the residence time matrix is $\mathbf{P}_{I I}$.

effectiveness if the coverage is $30 \%$ in 5 months, the reported cases are higher in comparison with the reduction achieved by restriction of mobility. On the other hand, if the $30 \%$ coverage is achieved very fast (2 months) and vaccine effectiveness is $80 \%$, the reduction in the number of cases is greater than the one obtained by restriction of mobility (see Fig. 5 (c)). In summary, the reduction of mobility among patches play a significant role in the mitigation of the disease close to the effect of immunization coverage of $30 \%$ achieved in 4 months. Finally, note that for the ranges explored in Fig. 5, it seems that coverage and the time needed to achieved such coverage has more impact than vaccine efficacy in disease control.

\section{Global sensitivity analysis}

To have a better understanding of how vaccine-associated parameters affect model outcomes, we perform a variance-based global sensitivity analysis (GSA) using the Sobol method [26]. In the presence of uncertainty in parameter values, GSA becomes an important methodology to quantify the sensitivity of model outcomes with respect to specific parameters as input factors. Within the Sobol framework, we estimate first-order indices to measure the contribution by a single parameter alone and total-order indices that include 
first-order effects but also all higher-order interactions. In this study, we investigate a range of vaccination scenarios varying coverage (20\%-60\%), delivery time (1-5 months), symptomatic fraction in the vaccinated-susceptible class $(0 \%-50 \%)$, duration of vaccine-induced immunity (6-12 months), and vaccine efficacy to prevent infection $(70 \%-95 \%)$. For the experiments, we varied the corresponding vaccine parameters within the proposed ranges for 5000 trials. During each trial, we randomly picked the value of each input parameter from the specified range and ran the model using those values. The outcomes of interest are the percentage reduction in SARS-CoV-2 cases in the asymptomatic, symptomatic, and reported infected classes in each patch compared with the no vaccination case for the year 2021.

Results of the GSA are shown in Fig. 6 as histograms in which the $x$-axis corresponds to the percentage reduction in the corresponding infected class and the $y$-axis is the bin's frequency. The first row shows the percentage reduction in the asymptomatic classes, the middle row in the symptomatic classes, and the bottom row in the reported classes. The histograms in blue, yellow, and green correspond to the patches $P 1, P 2$, and $P 3$, respectively. From the histograms, one can observe that the overall reduction for all patches and infected classes is close to $40 \%$ and the expected reduction in SARS-CoV-2 cases will be between $30 \%-50 \%$ for the parameters proposed in this work.

Sobol's first and total indices are obtained for all the asymptomatic classes $\left(\sum_{i} A_{i}\right)$ in Fig. $7(\mathrm{a})$, the symptomatic classes $\left(\sum_{i} I_{i}\right)$ in Fig. $7(\mathrm{~b})$ and the reported classes $\left(\sum_{i} I_{i}^{r}\right)$ in Fig. 7 (c). The $x$-axis corresponds to vector parameters $x=\left(x_{1}, x_{2}, x_{3}\right)$ with $x \in\{\theta, T, \psi, C, \tilde{\rho}\}$ and the vertical black lines represent $95 \%$ confidence intervals. The sensitivity analysis indicates that variations on vaccination coverage contribute the most to the overall variance of reduction of the number of SARS-CoV-2 cases. Vaccine efficacy and the time needed to achieve the vaccination coverage also play a significant role in the model outcomes. The duration of vaccine-induced immunity and the reduction in symptomatic disease seems to have little impact on the model behavior. This confirm the results obtained in section 4 . Finally, observe that the indices practically do not change among the infected classes.

\section{Discussion}

Less than a year after its emergence, the SARS-CoV-2 has taken more than one million lives and several countries are still struggling to maintain 
medRxiv preprint doi: https://doi.org/10.1101/2020.12.09.20246538; this version posted December 11, 2020. The copyright holder for this preprint (which was not certified by peer review) is the author/funder, who has granted medRxiv a license to display the preprint in perpetuity.

It is made available under a CC-BY-NC-ND 4.0 International license .

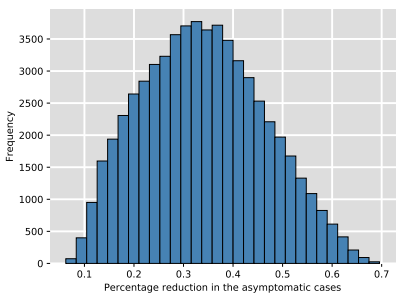

(a) Patch 1 asymptomatic

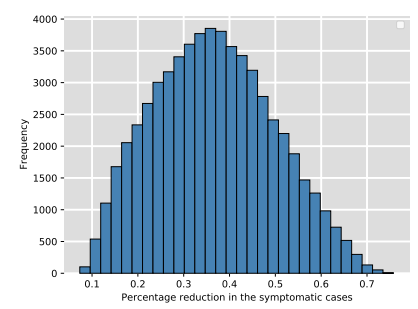

(d) Patch 1 symptomatic

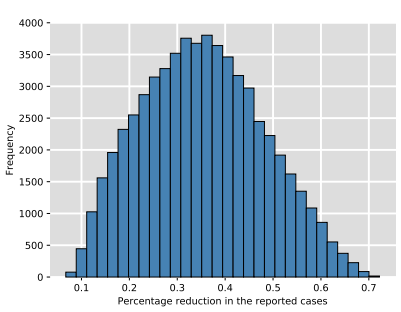

(g) Patch 1 reported

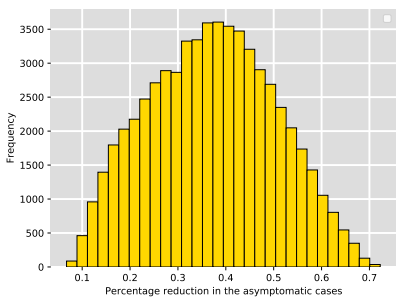

(b) Patch 2 asymptomatic

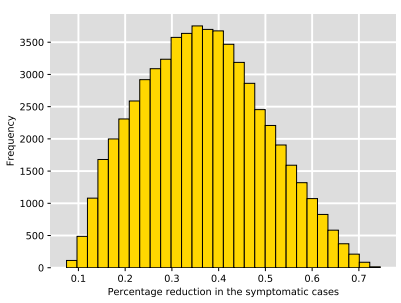

(e) Patch 2 symptomatic

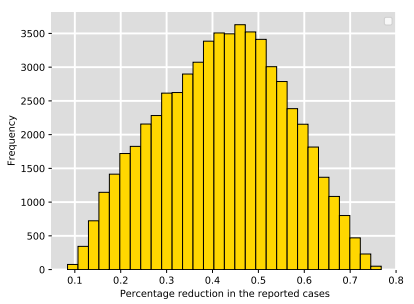

(h) Patch 2 reported

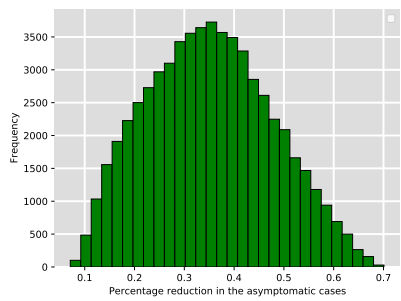

(c) Patch 3 asymptomatic

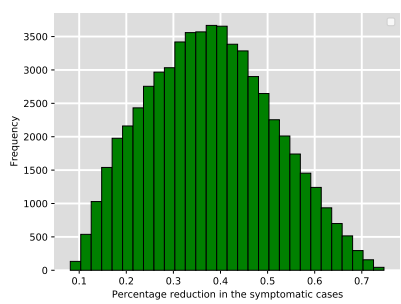

(f) Patch 3 symptomatic

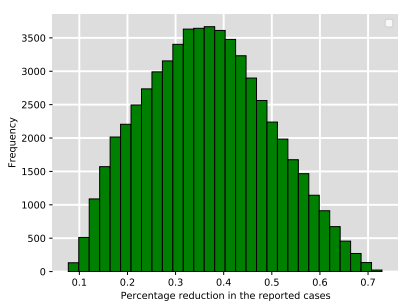

(i) Patch 3 reported

Figure 6: (First row) Percentage reduction in the asymptomatic classes. (Middle row) Percentage reduction in the symptomatic classes. (Bottom row) Percentage reduction in the reported classes. The histograms in blue, yellow and green correspond to the patches $P 1, P 2$ and $P 3$, respectively. The $x$-axis corresponds to the percentage reduction in the corresponding infected class and the $y$-axis is the bin's frequency. 


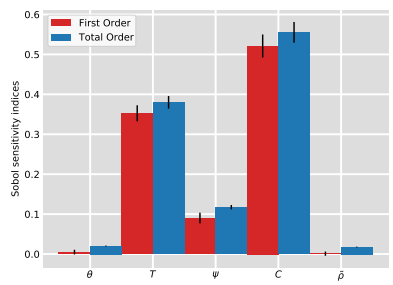

(a)

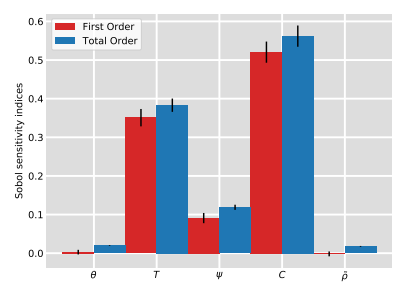

(b)

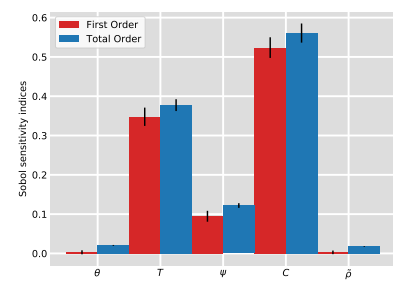

(c)

Figure 7: First and total order Sobol sensitivity indices with respect to the vaccine associated parameters for the mean percentage reduction in: (a) the asymptomatic classes $\left(\sum_{i} A_{i}\right),(\mathrm{b})$ the symptomatic classes $\left(\sum_{i} I_{i}\right)$, and (c) and the reported classes $\left(\sum_{i} I_{i}^{r}\right)$. The $x$-axis corresponds to vector parameters $x=\left(x_{1}, x_{2}, x_{3}\right)$ with $x \in\{\theta, T, \psi, C, \tilde{\rho}\}$ and the vertical black lines represent $95 \%$ confidence intervals.

the number of infections under control. At the beginning of the pandemic, in the absence of a vaccine, lockdowns, social distancing, and other nonpharmaceutical interventions have helped to slow the spread of the virus. However, the prolonged implementation of lockdowns at a national level has caused economic and psychological distress for many citizens, especially in marginal groups and large regions in the developing world. The introduction of an effective and safe vaccine represents a powerful weapon to fight the pandemic and the hope for a return to normality. The encouraging results presented from leading vaccine developers in November 2020 are good news. However, the vaccine efficacy, protective time-span and coverage needed to obtain a quick and significant reduction in the number of COVID-19 infections may be difficult to achieve. Hence, to aptly manage expectations once the vaccines become available in the global market, it is important to analyze to what extent the initial deployment of the vaccine will help to control the spread of disease.

In this work, we investigate the theoretical population-level impact of introducing a vaccine across several scenarios of interest. The analysis is based on a three patch risk-structured metapopulation epidemic model where patch infection prevlence depends on local environmental risk and intearctions between connected patches. In each patch, the dynamics are governed by a Kermack-McKendrick-type model and the connection among patches is described by a residence-time matrix. Unlike several previous COVID-19 epidemic models, our system allows the possibility of reinfections and incor- 
porates a vaccine that not only reduces susceptibility to infection but also prevents symptomatic disease. We explored how vaccination coverage, efficacy, and delivery time affect the control of the transmission dynamics in comparison with the restriction of mobility. Our results show that an efficacy of $80 \%$, with $40 \%$ coverage achieved in 5 months has a similar effect in comparison with restricted mobility without vaccination. The simulations also suggest that even with $90 \%$ vaccine effectiveness if the coverage is $30 \%$ in 5 months, the reported cases are higher in comparison with the reduction achieved by restriction of mobility. Concisely, the reduction of mobility among patches plays a significant role in the mitigation of the disease close in performance to the effect of immunization coverage of $30 \%$ achieved in 4 months. Our model also presents some projections on the number of reported cases in Mexico City as a function of relevant model parameters and specific vaccine conditions. Parameter values are constantly adjusting as more data become available and thus, these simulations are intended to explore plausible scenarios that can be of help for public health planning. They do not constitute quantitative predictions.

Considering the uncertainty associated to vaccine parameter values, we performed a global sensitivity analysis via Sobol's method. For our simulations, we varied the immunization coverage, delivery time, symptomatic fraction in the vaccinated-susceptible class, duration of vaccine-induced immunity, and vaccine efficacy to prevent infection. Our results suggest that if public health authorities can achieve an immunization coverage between 20\%$50 \%$ in the first half of the year 2021 with a vaccine of effectiveness higher than $70 \%$, the percentage reduction in the total number of SARS-CoV-2 infections in Mexico City is between 30\%-50\% by the end of 2021 in comparison with the no vaccination scenario. Furthermore, if there is restricted mobility, the simulations suggest that for a vaccine efficacy of $90 \%$ (or higher) and coverage above $30 \%$ before June 2021, then a very low number of COVID-19 infections are expected by the end of 2021. The computational experiments of the sensitivity analysis also imply that variations on vaccination coverage and the time needed to achieve such coverage are the most important drivers in the reduction of the number of SARS-CoV-2 cases. Vaccine efficacy plays a significant role, whereas the duration of vaccine-induced immunity and the reduction in symptomatic disease seem to have little impact on the global model behavior. As future work, we plan to extend our analysis to devise optimal vaccine allocation per age-group to minimize deaths and symptomatic COVID-19 infections. 


\section{Acknowledgements}

We acknowledge support from DGAPA-PAPIIT-UNAM grants IV 100220 (proyecto especial COVID-19) and IN115720. We thank the Mexican Federal Health Secretary and the WHO Strategic Advisory Group of Experts (SAGE) on immunization working group on COVID-19 vaccines for facilitating relevant data and policy recommendations.

\section{References}

[1] Acuna-Zegarra, M. A., Diaz-Infante, S., Baca-Carrasco, D., and Liceaga, D. O. (2020). Covid-19 optimal vaccination policies: a modeling study on efficacy, natural and vaccine-induced immunity responses. medRxiv.

[2] Acuña-Zegarra, M. A., Santana-Cibrian, M., and Velasco-Hernandez, J. X. (2020). Modeling behavioral change and covid-19 containment in mexico: A trade-off between lockdown and compliance. Mathematical Biosciences, page 108370.

[3] Bartsch, S. M., O’Shea, K. J., Ferguson, M. C., Bottazzi, M. E., Wedlock, P. T., Strych, U., McKinnell, J. A., Siegmund, S. S., Cox, S. N., Hotez, P. J., et al. (2020). Vaccine efficacy needed for a covid-19 coronavirus vaccine to prevent or stop an epidemic as the sole intervention. American journal of preventive medicine, 59(4):493-503.

[4] Bichara, D., Kang, Y., Castillo-Chavez, C., Horan, R., and Perrings, C. (2015). Sis and sir epidemic models under virtual dispersal. Bulletin of mathematical biology, 77(11):2004-2034.

[5] Bubar, K. M., Kissler, S. M., Lipsitch, M., Cobey, S., Grad, Y., and Larremore, D. B. (2020). Model-informed covid-19 vaccine prioritization strategies by age and serostatus. medRxiv.

[6] Buitrago-Garcia, D., Egli-Gany, D., Counotte, M. J., Hossmann, S., Imeri, H., Ipekci, A. M., Salanti, G., and Low, N. (2020). Occurrence and transmission potential of asymptomatic and presymptomatic sars-cov-2 infections: A living systematic review and meta-analysis. PLoS medicine, 17(9):e1003346. 
[7] Buonomo, B. (2020). Effects of information-dependent vaccination behavior on coronavirus outbreak: insights from a siri model. Ricerche di Matematica, page 1.

[8] Byambasuren, O., Cardona, M., Bell, K., Clark, J., McLaws, M.-L., and Glasziou, P. (2020). Estimating the extent of true asymptomatic covid19 and its potential for community transmission: systematic review and meta-analysis. Available at SSRN 3586675.

[9] Callaway, E. (2020a). Covid vaccine excitement builds as moderna reports third positive result. urlhttps://www.nature.com/articles/d41586020-03248-7. Accessed 16-11-2020.

[10] Callaway, E. (2020b). Russia announces positive covid-vaccine results from controversial trial. urlhttps://www.nature.com/articles/d41586-02003209-0. Accessed 11-11-2020.

[11] Callaway, E. (2020c). What pfizer's landmark covid vaccine results mean for the pandemic. urlhttps://www.nature.com/articles/d41586-020-031668. Accessed 09-11-2020.

[12] Castillo-Chavez, C., Bichara, D., and Morin, B. R. (2016). Perspectives on the role of mobility, behavior, and time scales in the spread of diseases. Proceedings of the National Academy of Sciences, 113(51):14582-14588.

[13] Delamater, P. L., Street, E. J., Leslie, T. F., Yang, Y. T., and Jacobsen, K. H. (2019). Complexity of the basic reproduction number (r0). Emerging infectious diseases, 25(1):1.

[14] Diekmann, O., Heesterbeek, J. A. P., and Metz, J. A. (1990). On the definition and the computation of the basic reproduction ratio $\mathrm{r} 0$ in models for infectious diseases in heterogeneous populations. Journal of mathematical biology, 28(4):365-382.

[15] Edridge, A. W., Kaczorowska, J., Hoste, A. C., Bakker, M., Klein, M., Loens, K., Jebbink, M. F., Matser, A., Kinsella, C. M., Rueda, P., et al. (2020). Seasonal coronavirus protective immunity is short-lasting. Nature Medicine, pages 1-3.

[16] Eikenberry, S. E., Mancuso, M., Iboi, E., Phan, T., Eikenberry, K., Kuang, Y., Kostelich, E., and Gumel, A. B. (2020). To mask or not to 
mask: Modeling the potential for face mask use by the general public to curtail the covid-19 pandemic. Infectious Disease Modelling.

[17] Gumel, A. (2012). Causes of backward bifurcations in some epidemiological models. Journal of Mathematical Analysis and Applications, 395(1):355-365.

[18] Hethcote, H. W. (2000). The mathematics of infectious diseases. SIAM review, $42(4): 599-653$.

[19] Iboi, E. A., Ngonghala, C. N., and Gumel, A. B. (2020). Will an imperfect vaccine curtail the covid-19 pandemic in the u.s.? Infectious Disease Modelling, 5:510 - 524.

[20] Le, T. T., Cramer, J. P., Chen, R., and Mayhew, S. (2020). Evolution of the covid-19 vaccine development landscape. Nat Rev Drug Discov, 19(10):667-8.

[21] Mena, R. H., Velasco-Hernandez, J. X., Mantilla-Beniers, N. B., Carranco-Sapiéns, G. A., Benet, L., Boyer, D., and Castillo, I. P. (2020). Using the posterior predictive distribution to analyse epidemic models: Covid-19 in mexico city. arXiv preprint arXiv:2005.02294.

[22] Ngonghala, C. N., Iboi, E., Eikenberry, S., Scotch, M., MacIntyre, C. R., Bonds, M. H., and Gumel, A. B. (2020). Mathematical assessment of the impact of non-pharmaceutical interventions on curtailing the 2019 novel coronavirus. Mathematical Biosciences, page 108364.

[23] NYT (2020). Astrazeneca's coronavirus vaccine, easy and cheap to produce, appears effective. urlhttps://www.nytimes.com/2020/11/23/business/astrazeneca-oxfordcoronavirus-vaccine.html. Accessed 25-11-2020.

[24] Reuters (2020). Mexico signs deal with pfizer for 34.4 million doses of covid-19 vaccine. https://www.reuters.com/article/us-health-coronavirusmexico/mexico-signs-deal-with-pfizer-for-34-4-million-doses-of-covid-19vaccine-idUKKBN28C2VK.

[25] Saldaña, F., Flores-Arguedas, H., Camacho-Gutiérrez, J. A., and Barradas, I. (2020). Modeling the transmission dynamics and the impact of the control interventions for the covid-19 epidemic outbreak. 
[26] Saltelli, A., Ratto, M., Andres, T., Campolongo, F., Cariboni, J., Gatelli, D., Saisana, M., and Tarantola, S. (2008). Global sensitivity analysis: the primer. John Wiley \& Sons.

[27] Salud, S. (2020). Datos covid-19 méxico. urlhttps://datos.covid19.conacyt.mx/. Accessed 12-11-2020.

[28] Santamaría-Holek, I. and Castaño, V. (2020). Possible fates of the spread of sars-cov-2 in the mexican context. Royal Society open science, $7(9): 200886$.

[29] Truscott, J. and Ferguson, N. M. (2012). Evaluating the adequacy of gravity models as a description of human mobility for epidemic modelling. PLoS Comput Biol, 8(10):e1002699.

[30] Van den Driessche, P. and Watmough, J. (2002). Reproduction numbers and sub-threshold endemic equilibria for compartmental models of disease transmission. Mathematical biosciences, 180(1-2):29-48.

[31] WHO (2020). Coronavirus disease (covid-19): Weekly epidemiological update (17 november 2020). urlhttps://reliefweb.int/report/world/coronavirus-disease-covid-19weekly-epidemiological-update-17-november-2020. Accessed 17-11-2020.

[32] Zhao, H. and Feng, Z. (2020). Staggered release policies for covid-19 control: Costs and benefits of relaxing restrictions by age and risk. Mathematical biosciences, 326:108405. 